AperTO - Archivio Istituzionale Open Access dell'Università di Torino

Methods to Retrieve the Cloud-Top Height in the Frame of the JEM-EUSO Mission

This is a pre print version of the following article:

Original Citation:

Availability:

This version is available http://hdl.handle.net/2318/1694897

since 2019-03-16T14:35:59Z

Published version:

DOI:10.1109/TGRS.2018.2854296

Terms of use:

Open Access

Anyone can freely access the full text of works made available as "Open Access". Works made available under a Creative Commons license can be used according to the terms and conditions of said license. Use of all other works requires consent of the right holder (author or publisher) if not exempted from copyright protection by the applicable law. 


\title{
Methods to retrieve the Cloud Top Height in the frame of the JEM-EUSO mission
}

\author{
A. Anzalone, M. Bertaina, S. Briz, C. Cassardo, R. Cremonini, A. J. de Castro, \\ S. Ferrarese, F. Isgrò, F. López, I. Tabone
}

\begin{abstract}
The JEM-EUSO (Japanese Experiment ModuleExtreme Universe Space Observatory) telescope will measure Ultra High Energy Cosmic Ray properties by detecting the UV fluorescence light generated in the interaction between cosmic rays and the atmosphere. Therefore, information on the state of clouds in the atmosphere is crucial for a proper interpretation of the data. For a real-time observation of the clouds in the telescope Field of View (FoV), JEM-EUSO will use an atmospheric monitoring system composed of a Lidar (LIght Detection And Ranging) and an Infra-Red Camera. In this article the focus is on the IR camera data. To retrieve the Cloud Top Height (CTH) from IR images, three different methods are considered here. The first one is based on bi-spectral stereo vision algorithms and requires two different views of the same scene in different spectral bands. For the second one, brightness temperatures provided by the IR camera are converted to effective cloud top temperatures, from which the CTH is estimated using the vertical temperature profiles. A third method that uses primary Numerical Weather Prediction (NWP) model output parameters, such as the cloud fraction, has also been considered to retrieve the CTH. This article presents a first analysis, in which the heights retrieved by these three methodologies are compared with the heights given by MODIS (MODerate resolution Imaging Spectroradiometer) sensor installed on the polar satellite Terra. Since all these methods are suitable for the JEM-EUSO mission, they could be used in the future in a complementary way to improve the accuracy of the CTH retrieval.
\end{abstract}

\section{INTRODUCTION}

The flux of Extreme Energy Cosmic Rays (EECRs), which are cosmic rays with an energy above $5 \times 10^{19} \mathrm{eV}$, is of the order of 1 particle/ $\mathrm{km}^{2} /$ century. This makes their detection challenging. Currently, the giant ground-based detectors, such as the Pierre Auger Observatory [1], [2], cannot detect more than $\sim 30$ events/year in this energy range.

The space-based observation of EECRs, by means of telescopes on dedicated satellites or on-board the International Space Station (ISS), provides the means to increase the collection power of such cosmic rays by at least one order of magnitude. Among the ideas presented in the past, the JEMEUSO concept [3], [4] is the most advanced from both the programmatic and technological point of view.

Anna Anzalone is with INAF - IASF, Istituto di Astrofisica Spaziale e Fisica Cosmica, Palermo and INFN -Sezione di Catania, Italy

M. Bertaina, C. Cassardo, R. Cremonini, S. Ferrarese and I. Tabone are with Dipartimento di Fisica, Università degli Studi di Torino and INFN - Sezione di Torino, Italy

S. Briz, A. J de Castro and F. López are with Universidad Carlos III de Madrid, Avda. de la Universidad 30, 28911 Leganés, Spain

F. Isgrò is with Dipartimento di Ingegneria Elettrica e delle Tecnologie dell'Informazione, Università degli Studi di Napoli Federico II and INFN Sezione di Napoli, Italy
When an EECR particle reaches the atmosphere, it produces a nuclear interaction, which leads to the generation of a cascade of billions of particles that form a so-called Extensive Air Shower (EAS). The electrons, continuously produced during the evolution of the cascade in the atmosphere, excite the nitrogen molecules in air and produce fluorescence light in the spectral band $300-400 \mathrm{~nm}$. The cascade starts as a very faint signal at the altitude of $30-40 \mathrm{~km}$, depending on the direction of the incoming EECR, and reaches its maximum in terms of light intensity at 5-10 km above the Earth's surface. The observational principle of the JEM-EUSO telescope is to record the time evolution and the light intensity of such EAS cascades. The space-based observation requires specific data treatment regarding the presence of clouds that might distort or prevent the detection of the EAS cascade in the atmosphere. In the case of JEM-EUSO, the ISS is moving with a sub-satellite point speed of $\sim 7.5 \mathrm{~km} / \mathrm{sec}$ and an orbital inclination of $51.5^{\circ}$ around the Earth, therefore the JEM-EUSO telescope will encounter a range of meteorological and cloud conditions in the FoV with changes on short timescales. The influence of clouds is obviously dependent on their top altitude and depth. In general, to correctly analyse the EAS, the CTH must be determined with an accuracy of $500 \mathrm{~m}$. If the CTH is calculated from its temperature, the latter should be determined with an accuracy of $3 \mathrm{~K}$, in order to vertically localize the $\mathrm{CTH}$ with a sufficient accuracy for EECR detection.

There are already many meteorological satellites providing observations of atmospheric and cloud conditions from multi-spectral observations with good spatial and temporal resolution, but the JEM-EUSO mission requires spatially and temporally simultaneous measurements of these atmospheric parameters. Therefore, a specific instrument is necessary to detect synchronized atmospheric information and cloud coverage in the FoV of the UV telescope.

For this reason, JEM-EUSO will be equipped with an Atmospheric Monitoring System (AMS), see Figure 1, which will include: a) an InfraRed (IR) camera; b) a Lidar device. Moreover it will be supported by global atmospheric models generated from the analysis of all available meteorological data provided by global weather services, such as the European Centre for Medium-range Weather Forecasts (ECMWF) (http://www.ecmwf.int/) [5]. Lidar data provide precise measurements of atmospheric parameters, such as cloud optical depth, cloud height etc., in certain localised points in the FoV (i.e., around the EAS location). Whereas to monitor atmospheric conditions in the whole JEM-EUSO telescope FoV, an imaging sensor, such as an IR camera, is required. This 


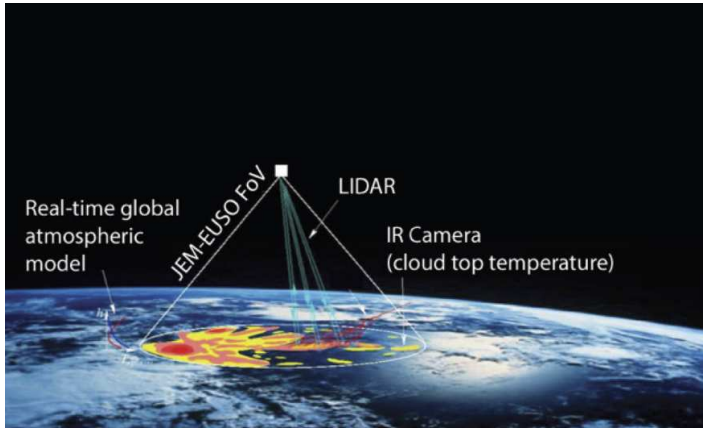

Fig. 1. Sketch of the Atmospheric Monitoring system of the JEM-EUSO mission.

is particularly important in order to understand the fraction of time in which the JEM-EUSO sensor is unable to detect EAS because of the presence of clouds. Therefore these systems are complementary to each other and provide useful measurements. In this paper the attention is focused on the IR camera. Its $\mathrm{FoV}$ is $48^{\circ}$ and the Instantaneous $\mathrm{FoV}^{1}$ is $0.08^{\circ}$, while the spectral range is $8-14 \mu \mathrm{m}$, centred at the IR band. However, to retrieve more information about the IR conditions, this spectral range is split into two spectral sub-bands centred at $10.8 \mu \mathrm{m}$ and $12 \mu \mathrm{m}$ and having a spectral width of $\sim 1$ $\mu \mathrm{m}$ each. More information about the current design of the IR camera and its requirements can be found in [6].

The objective of this work is to study the feasibility of different strategies to retrieve the CTH from the JEM-EUSO IR Camera images. Three methods are considered in this work: stereo vision algorithms, radiometric methods and numerical weather prediction models. The first requires two different views of the same scene. The second is based on the relationship between the Cloud Top Temperature (CTT) and the CTH. In this case, the Brightness Temperature (BT) in two spectral bands is required to retrieve the CTT. The third is a more novel technique based on the use of a meteorological forecasting model. Specifically the CTH is retrieved from the cloud fraction simulated by the Weather Research and Forecasting model (WRF).

To test these methods on real data, different cloudy scenes located over the Atlantic Ocean near the African coasts have been analysed. These scenes were chosen for several reasons.

As the area was scanned by the Meteosat-9 (MSG2) and Meteosat-10 (MSG3) satellites and Envisat/AATSR (Environmental Satellite/Advanced Along-Track Scanning Radiometer), stereo vision can be applied. Since the same area is observed by the Terra satellite, MODIS (MODerate resolution Imaging Spectroradiometer on board the Terra and Aqua satellites [7]), IR images can be used for the radiative methods. Furthermore, both MSG, MODIS and AATSR provide data in the same bands as the JEM-EUSO IR camera. In addition, the resulting CTHs can be reasonably compared to the MODIS referential height because the scans are almost simultaneous. Since the JEM-EUSO telescope will spend most of the time viewing oceans, these scenes reproduce typical working con-

\footnotetext{
${ }^{1}$ The IFoV is meant to be the angle subtended by a single detector element on the axis of the optical system
}

ditions of the mission. The scenes are close to the equatorial zone, so the geostationary satellite's nadir resolution is roughly maintained within the whole region and high quality images are available. Because the scenes are close to a radiosounding station, actual vertical profiles close in time to the satellite observations could be used with the radiative methods. Finally, because the set of scenes selected (see table I) is an appropriate testing bench since it involves different types of clouds: both liquid water and ice clouds and thick/thin clouds. In table I (page 7) the percentages of those kinds of clouds are detailed where thick cloud means optical thickness higher than 2 and thin corresponds to optical thickness lower than 2. These percentages have been calculated with the phase and optical thickness cloud products provided by the MODIS collection 6, MOD06, [8].

Four additional scenarios were studied to consider the effect of different latitudes in different seasons. Since the ISS orbit has an inclination of $51.5^{\circ}$, only middle latitude images have been analyzed. Moreover, these images have been also selected over oceans because EECR detection can be performed only on dark regions, unpolluted by natural or artificial lights. Therefore, oceans represent $\sim 80 \%$ of the total available time all along the ISS orbits. The three methods presented here were developed and tested on these study cases.

\section{Methods}

\section{A. Stereo Method}

During the last twenty years, space-based stereoscopic methods have been raising interest among researchers and are improving thanks to new satellite-based instruments [9], [10], [11], [12], [13], [14], [15]. Stereo reconstruction is an alternative way to estimate the $\mathrm{CTH}$ compared with the more common radiative methods. Whilst an accurate radiometric calibration and the knowledge of extra atmospheric parameters are fundamental for the traditional methods, the main requirements of this approach are an accurate image analysis, a proper setup of the stereo system and the knowledge of the system's geometric parameters. The 3D structure of an object in a standard binocular vision system ([16], [17]), is inferred from the analysis of the two images acquired by spatially separated cameras. Estimation of the resulting parallax effect contributes to the reconstruction of the distance of the object from the visual sensor, or its depth ([18]). In remote sensing observations, measurements of the CTH can be produced both by geostationary and polar satellites. Geostationary orbiters can be coupled in such a way that the intersection of both FoVs provides quasi-synchronous stereo pairs of the same scene, while some polar satellites are already equipped with instruments with stereo capability such as Envisat/AATSR, Sentinel3/SLSTR (Sea and Land Surface Temperature Radiometer), Terra/MISR (Multi-angle Imaging SpectroRadiometer).

In the JEM-EUSO mission, the AMS includes an Infra-Red camera that can also be used as a stereo sensor, despite its monocular nature. In fact, exploiting the movement of the ISS, it is possible to acquire two consecutive observations at two different and relatively close instants, in such a way that part of the images overlap. These two images can be considered 


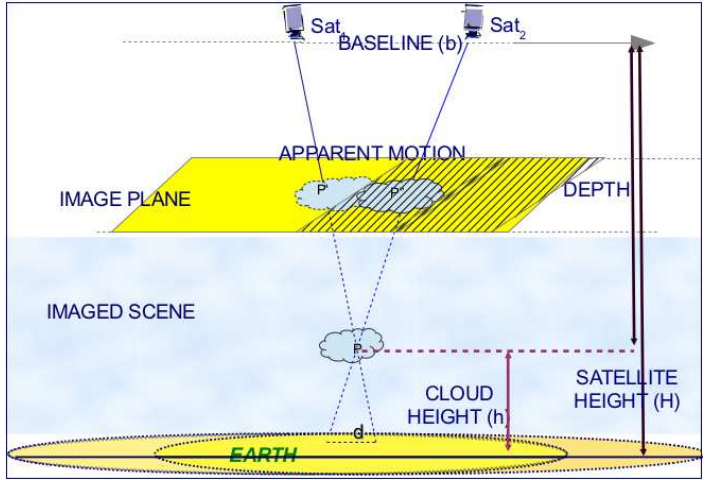

Fig. 2. Stereo Reconstruction. The scheme shows two corresponding points $\left(P^{\prime}\right.$ and $P^{\prime \prime}$ ) detected on the images in the matching step from the retrieved disparity, and the 3D point $P$ of which they are the projections, recovered by triangulating the optical rays. The stereo bi-band acquisition is highlighted by the grid/no-grid on the cloud

as a stereo pair for the same scene observed from different viewing angles. Moreover, the camera will use two different bands for the observations, hence, for each orbit of the ISS, all the scenes in the FoV of the camera will be represented by multiband stereo pairs.

The CTH retrieval procedure is mainly composed of two steps that allow the determination of the optical rays and the depth (Figure 2). The matching step identifies the same features in both members of the image pair, and estimates for each pixel the parallax effect. In general, this is visible as an apparent displacement of the cloud from one view to the other, called disparity, that is measured in pixels and depends on the object distance. The time lag between two consecutive observations determines the distance between the two viewpoints, that is known as baseline of the stereo system.

Finally the 3-D coordinates of a cloudy point are estimated by projecting the optical rays backwards. These are obtained from the detected points of correspondence between the two images and the satellite positions at the time of acquisition (Figure 2).

1) Stereo Algorithm: Due to the current design of the JEMEUSO IR camera, a multispectral stereo approach must be considered instead of the more common single band appraoch. For this reason, in this work a refined version [19] of the multi band stereo method originally proposed in [20] has been used. The algorithm has been applied to geostationary MSG/SEVIRI (Meteosat Second Generation/Spinning Enhanced Visible and InfraRed Imager [21]) and polar Envisat/AATSR (Environmental Satellite/Advanced Along-Track Scanning Radiometer [22]) satellite data, that are described in section III-A.

The method is a multispectral stereo algorithm based on the idea that the height can be estimated using a coarser resolution than a single pixel, exploiting the fact that, in limited regions, pixels showing the same temperature are very likely to be located at the same altitude. For each member of a stereo pair, the range of the pixel BTs is evenly split into sub intervals. On the images, this is used to determine separated areas that include pixels having values between the lower and upper limits of each interval. In this way the images are partitioned into regions corresponding to the selected temperatures. The

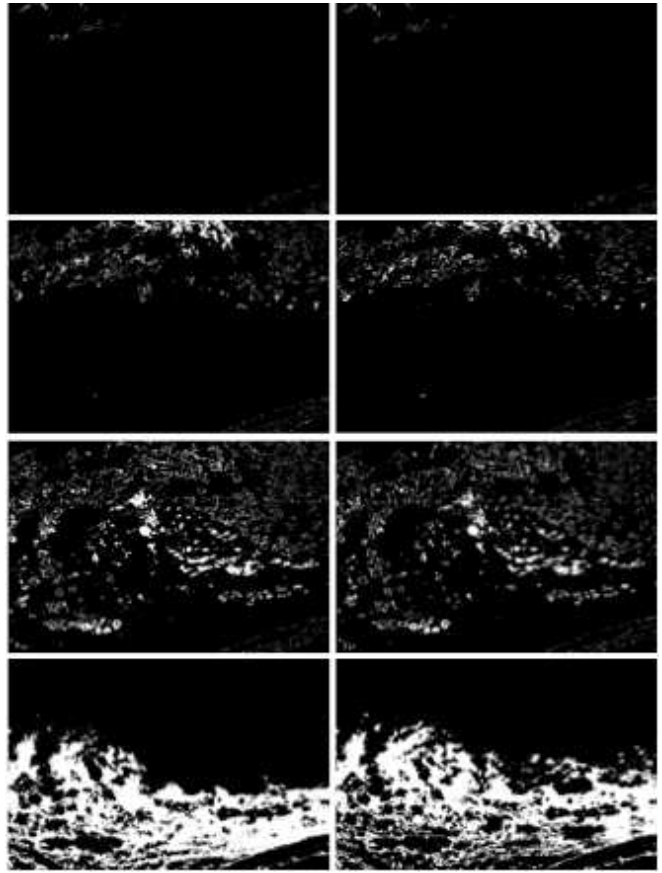

Fig. 3. Segmentation. Example of binary masks: on the left column, masks from a $10.8-\mu \mathrm{m}$ image and on the right, masks from a $12-\mu \mathrm{m}$ image. From top to bottom, masks corresponding from the coldest to the warmest sub ranges of temperatures.

width of the sub intervals is experimentally fixed. Each region generates a binary mask $M$ (see Figure 3 ) of the same size of the original image I, where, for each pixel $p, M(p)=1$ if $I(p)$ belongs to the fixed sub interval, otherwise $\mathrm{M}(\mathrm{p})=0$.

For each pair of corresponding masks between the two images, the disparity is determined as the geometrical translation applied to the mask in the first image and maximising the overlap between the two masks. The disparity retrieved for the whole region, is assigned to each pixel of the first image belonging to the region. The role of the two images is then swapped, so that two disparity maps are reconstructed: $d_{12}$ from the first image to the second one, and $d_{21}$ from the second image to the first one. The resulting disparity maps are then tested for the possible dissimilarities using a consistency check method, widely used in stereo matching. The final disparity $d$ is assigned as follows for each pixel p:

$$
\mathrm{d}(\mathrm{p})= \begin{cases}\mathrm{d}_{12} & \text { if } \operatorname{abs}\left(\mathrm{d}_{12}-\mathrm{d}_{21}\right)<\mathrm{t} \\ \text { not assigned } & \text { otherwise }\end{cases}
$$

where $t$ is a threshold value, meaning that the error tolerated in the disparity, must be not larger than $t$ pixels. For this study, a height value is not assigned to those pixels that do not satisfy the consistency test. Finally, the pixel depths are calculated by triangulation: to intersect the optical rays, ancillary data provided by the satellite databases are used.

For AATSR CTHs, the Prata\&Turner [10] formula (2) is applied:

$$
\mathrm{CTH}=\frac{\mathrm{d}}{\tan \left(\theta_{f}\right)-\tan \left(\theta_{n}\right)},
$$

where $d$ is the retrieved disparity along track, $\theta_{f}$ and $\theta_{n}$ are the viewing forward and nadir zenith angles at the Earth's surface, 
supplied by the satellite database.

In the case of MSG data, the zenith angles are not directly provided, hence, the two projections of the cloudy pixels and the satellite positions at the time of the acquisitions are used for evaluating the intersection of the optical rays. The former are given by the corresponding pair of matched points from the disparity, while the latter, are supplied by the database for each row of the images. The crossing point detection and consequently the final $\mathrm{CTH}$, will depend on uncertainties of the correct orbiter location, the geolocation precision, and the coupling made by the matches.

2) Data: in this paper we have considered a geostationary stereo system and stereo pairs of images provided by a polar satellite to test the method on different configurations, whose characteristics are briefly described here, focusing on the stereo set up.

The first system is given by the combination of the Meteosat/SEVIRI satellites MSG-2 and MSG-3, located on the Equatorial plane respectively at $9.5^{\circ} \mathrm{N}$ and $0^{\circ} \mathrm{E}$ at about 36,000 $\mathrm{km}$ of altitude. MSG-3 provides full disc imagery every 15 minutes of Europe and Africa, whereas the Rapid Scan System of MSG-2 delivers images every 5 minutes over the Northern Hemisphere. Europe, parts of Africa and adjacent oceans are then observed from two different viewpoints, and with a pixel resolution of $3 \mathrm{~km}$ at the Sub Satellite Point (SSP). The baseline of $\approx 7000 \mathrm{~km}$ between MSG-2 and MSG-3 positions, is insufficient to provide an accurate reconstruction for all CTHs. This occurs because Meteosat satellites were not designed to be a stereo system. Following [13], the accuracy of the CTH $\left(\sigma_{C T H}\right)$ estimated from a stereo system can be expressed as:

$$
\sigma_{\mathrm{CTH}}=\frac{\sigma_{\mathrm{d}}}{\mathrm{b} / \mathrm{H}_{\mathrm{s}}},
$$

where $\sigma_{d}$ is the disparity estimation error at the ground, $b$ is the baseline of the stereo system and $H_{s}$ is the satellite altitude. Using Equation 3 and considering an average disparity error of no more than half a pixel, i.e., $\sigma_{d}= \pm 1.5 \mathrm{~km}$, the accuracy results within $7.6 \mathrm{~km}$. Hence, if the disparity is estimated with an accuracy of half a pixel, in the Meteosat configuration clouds with heights $h \in[7.5,15] \mathrm{km}$ could be difficult to distinguish from each other and lower clouds could be merged into the background. The two imaging devices are quasi-synchronous, and ancillary data include also the actual satellite positions for each row of the images utilised for the CTH estimation.

The second stereo system is provided by the AATSR radiometer with dual viewing capability. It was on board the polar satellite Envisat at an altitude of $\approx 800 \mathrm{~km}$ with a velocity of $\approx 7.5 \mathrm{~km} / \mathrm{s}$. It collected data from 7 spectral bands (here we use those at 11 and $12 \mu \mathrm{m}$ ) making two observations of the same area: one along the direction of the orbit track, at an incidence angle of $55^{\circ}$ decreasing to $47^{\circ}$ at the edges of the swath, flying towards the scene (forward view). The second observation is taken after $120 \mathrm{~s}$, at a viewing angle close to $0^{\circ}$ (nadir view) at the centre of the swath and increasing to $22^{\circ}$ at the edges. Even in this case, the stereo system features are different from the JEM-EUSO ones, but closer than the MSG configuration. The Gridded BT products include geolocated data mapped into a $512 \times 512$ grid with $1 \mathrm{~km}$ of pixel resolution and resampled using the nearest neighbour method.

The accuracy of the CTH can be predicted, as for the MSG case, using Equation 3. Considering an average disparity of 1 pixel, the resulting accuracy $\sigma_{d}$ is in the range $\approx 0.8 \mathrm{~km}$ to $\approx 1.5 \mathrm{~km}$ depending on the across track position. This can be improved to between $\approx 0.4 \mathrm{~km}$ and $\approx 0.7 \mathrm{~km}$ if the disparity accuracy is half a pixel. It is worth pointing out that this measure only considers the geometry of the system, and it does not take into account other elements that can affect the final retrieval as highlighted in section III-A.

\section{B. WRF Cloud Fraction Method}

Numerical Weather Prediction (NWP) models simulate many primary atmospheric quantities which are useful for the the CTH estimation without involving satellite IR sensors.

In this study, the mesoscale Weather Research and Forecasting (WRF) model, Version 3.6, was used. WRF is a fully compressible, non-hydrostatic, primitive-equation model with multiple-nesting capabilities that can enhance resolution over the areas of interest. The main WRF parametrizations concern microphysics, long and short wave radiation, surface layer physics, boundary layer physics and cloud physics. A complete description of the WRF modelling system can be found in [23]. The model outputs are organized in three-dimensional grids at every time interval. The spatial and temporal resolutions are limited by the terrestrial data set resolution and the available computational resources.

The CTH retrieval methodology investigated here is based on the WRF simulated Cloud Fraction (CF), a variable expressing the fraction of cloudiness in a model grid box, which assumes values from 0 to 1 and is given for each model level. The basic idea is that each grid cell with a CF higher than a fixed threshold contains a cloud. Starting from the upper level of the considered WRF simulation, and going down through the troposphere, whenever a cloud is detected for the first time in the vertical, the height at which the grid cell has been identified as cloud can be assumed as the CTH. The result of this procedure is therefore a 2-D matrix of CTH. In this study, the threshold was experimentally set to 0.2 for all simulations.

1) Data: A WRF model simulation for each scene described by MODIS satellite was run. All simulations were set with a spatial resolution of $9 \mathrm{~km}, 40$ vertical levels (up to about $16 \mathrm{~km}$ ) and a time step of $60 \mathrm{~s}$. The model has been initialized with the ECMWF global model output data, released with a horizontal resolution of $0.125^{\circ} \times 0.125^{\circ}$ (longitude $\mathrm{x}$ latitude), while the main parametrization schemes selected were: Rapid Radiative Transfer Model [24] for longwave radiation, Dudhia scheme [25] for shortwave radiation, MM5 similarity model for surface layer, Yonsei University Scheme [26] for boundary layer, Morrison-Double-Moment [27] for microphysics and explicit for cumulus parametrization, suitable for cloud-resolving simulations.

\section{Radiative Methods}

The CTH retrieval can be performed using radiometric information of the cloud. The radiation emitted by the cloud 
is basically related to its emissivity and temperature according to the Planck's law. Therefore the radiation received by the IR camera could be used to get the CTT, and then to estimate the CTH by using the relationship between temperature and height. However, due to atmospheric effects (mainly gas absorption/emission), the IR radiance measured by the IR camera would not be exactly the one emitted by the cloud top. Hence the temperature retrieved from the measured radiance (BT) would not be the CTT. The radiative method consists of some corrective algorithms to account for the atmospheric effects retrieving the actual CTT from the BT, and to calculate the $\mathrm{CTH}$ from the CTT using the temperature vertical profiles provided by WRF model.

1) CTT retrieval algorithms: the objective of these algorithms is to retrieve the CTT from the cloud BT measured by the IR camera automatically and as independently as possible, i.e., without any ancillary information. Since the design of the IR camera allowed us to consider two spectral bands $(10.8-\mu \mathrm{m}$ and $12-\mu \mathrm{m}$ bands), the bi-spectral algorithms can be applied for the CTT retrieval. Some authors ([28], [29], [30]) have used two adjacent spectral bands to define algorithms able to retrieve the Earth's surface temperature, called Split-Window Algorithms (SWAs). These algorithms consider the target as a blackbody, i.e., a surface of effective emissivity $1(\epsilon=1)$, henceforth referred to as emissivity. The SWA developed in this work to retrieve the CTT is based on this hypothesis $(\epsilon=1)$, therefore it is not expected to retrieve the CTT of thin clouds $(\epsilon<1)$ accurately. Although many clouds can be considered a blackbody (thick clouds), thin clouds are also significant and very important for EAS reconstruction. For these reasons, two approaches have been explored: a) a SWA and b) an algorithm based on a simplification of the Radiative Transfer Equation (RTE).

a) Split Window Algorithm (SWA) (for thick clouds): A detailed description of the method applied here can be found in [31]. This method has been validated for a wide set of scenarios simulated using the well-known MODerate resolution atmospheric TRANsmission radiative code (MODTRAN, [32]). Simulations were performed for a range of different values for the vertical profile of the water vapor mixing ratio, height and optical thickness of the cloud. In this theoretical study, retrieval errors in the CTT of $0.3 \mathrm{~K}$ were found for the worst scenario (low clouds in humid atmospheres). When applied to real scenarios (taken from MODIS images), this SWA gives very accurate CTT for low thick clouds (mostly liquid water clouds), being the error lower than $1 \mathrm{~K}$ in $80 \%$ of these situations.

b) Algorithms to retrieve the CTT (for thin clouds): If the cloud is optically thin, it is necessary to retrieve not only the temperature but also the emissivity. The procedure described in this section is applied for thin clouds and involves two kinds of algorithms, one for retrieving the emissivity and another one for calculating the CTT. To retrieve the emissivity two different algorithms have been studied: one based on Look Up Tables (LUTs), that exploits the relationship between the BTDs and the emissivity, and an algorithm based directly on a simplification of the RTE.

One of the commonly used approaches to retrieve the emissivity uses LUTs [33]. To build the LUTs, the BTs in the JEM-EUSO spectral bands have been calculated from radiances obtained with the MODTRAN code for different scenarios (different atmospheric conditions and clouds and different land surface temperatures). The LUTs are based on the relationship between the difference in the BT values in both bands (Brightness Temperature Difference-BTD) and the cloud emissivities used in the simulations for different CTTs. To retrieve the emissivity of the real scenarios the measured BTDs were entered in the LUT previously calculated from the simulations. The LUT-based procedure was also validated with simulations (corresponding to other set of atmospheric conditions and cloud types) and checked in real scenarios (MODIS images). The main result of the study is that the error increases as the emissivity decreases. In real scenarios, the differences between the emissivity retrieved by the LUT and the one provided by MODIS (used as the true value) was sometimes higher than $40 \%$ for emissivities lower than 0.5 . Many factors contribute to the errors of the temperature and emissivity retrievals [34], and many of them are related to the nature of the physics itself. For this reason, the retrieval of the cloud emissivity by using the LUT procedure is only suitable for thin clouds with emissivities in the range $0.5<\epsilon<1$.

Due to the limitations of the LUT procedure, a second approach was developed. This approach is based on two simplifications of the RTE explained in [31]. The first simplification aims at eliminating the $\mathrm{CTH}$ from the equation as it is one of the unknowns. This step requires knowledge of the surface temperature, the radiance emitted by the atmosphere and the atmospheric transmittance. To automatise the process, these data are not calculated for the specific atmospheric conditions of each image. The surface temperature used in this paper has been obtained from the Surface_Temperature included in the MODIS cloud product MOD06, [8]. The atmospheric radiance and transmittance are calculated running MODTRAN code with different atmospheric profiles to consider different latitudes and seasons. In the future, information provided by weather prediction models, such as WRF, could be used to calculate these values for the specific conditions of the scene. At this stage, where the methods were checked independently, we preferred not to use the WRF information for the emissivity and CTT retrievals, bearing in mind that the results of the radiative method can be improved in the future. The second simplification allowed us to obtain the emissivity from those radiances, with the simplifying assumption that the emissivity and the radiance emitted by the cloud were considered equal in both bands. The main conclusion of this study is that these simplifications are accurate only for high thin clouds. The physical reason of this behaviour is that lower atmospheric layers emit/absorb more radiation. Neglecting the atmosphere above the cloud in the RTE simplification is only valid if the cloud is high enough. As an example, the emissivity error in a theoretical cloud of emissivity 0.78 is $6 \%$ when located at $8.5 \mathrm{~km}$, and $27 \%$ when located at $5.5 \mathrm{~km}$. For that reason, this procedure can be only applied to high clouds. However, since low clouds rarely have small emissivities $(\epsilon<0.5)$, this assumption does not seem restrictive.

After retrieving the emissivity, the CTT was calculated with 


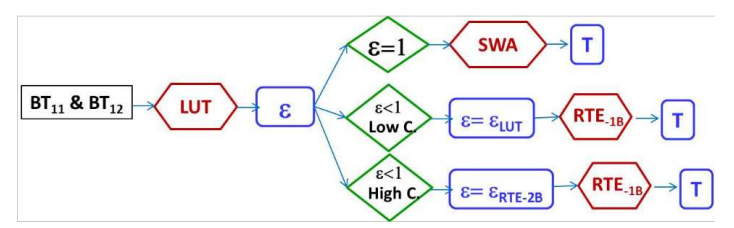

Fig. 4. Flow chart of the procedure to retrieve the CTT.

the equation obtained in the first simplification, applied to the $10.8-\mu \mathrm{m}$ band. This procedure was also checked with simulations, i.e., the BTs in both bands were calculated for different scenarios where atmospheric conditions, emissivity and CTT were known. The procedure explained above, was applied to retrieve the CTT and it was compared with the temperature used in the simulation. For example, the difference between the CTT used in the simulation and the retrieved temperature is $0.75 \mathrm{~K}$ when applied to clouds located at 8.5 $\mathrm{km}$, but it increases when the CTH decreases.

Considering the limitations of the SWA, the LUTs and the RTE approximations, a general procedure to retrieve the CTT is proposed: first, the LUT is applied to determine the emissivity. If $\epsilon=1$, then the SWA directly retrieves the CTT. If $0.5<\epsilon<1$, the LUT is applied to low clouds, and the RTE simplification in two bands is used to retrieve the emissivity of high clouds. Then, the RTE simplification is applied to BT in the $10.8-\mu \mathrm{m}$ band to retrieve the CTT (see Figure 4). The limit between low and high clouds concerns the retrieval procedure, not the standard cloud classification, and was defined here considering the height at which the radiative transfer equation simplification is valid. More precisely, the criterion to separate high/low thin clouds was established on the basis of the temperature accuracy. A conservative limit was set to $273 \mathrm{~K}$, at which the difference between the CTT in the simulations and the retrieved CTT is lower than $1 \mathrm{~K}$. This limit corresponds to a height of $4-5 \mathrm{~km}$ approximately, at the latitude of these particular scenarios.

2) $\mathrm{CTH}$ retrieval procedure: the objective of the $\mathrm{CTH}$ retrieval procedure is to calculate the $\mathrm{CTH}$ from the CTT by using the relation between temperature and height, contained in the vertical thermal profile of the atmosphere. Usually, this conversion is performed applying standard atmospheric profiles or observed radiosounding data. However, the former provides an oversimplified tropospheric vertical profile in which the temperature varies almost linearly with the height. Conversely, actual profiles are rare and available only few times per day, a rate that is too small considering the variability of the atmosphere. For these reasons more suitable profiles must be taken into account. This subsection illustrates the possibility of using temperature profiles simulated by NWP models, and specifically by the mesoscale model WRF.

WRF model output provides a temperature value for each grid-point and a sequence of horizontal and vertical interpolations ensures a temperature value for each pixel in the domain for each corresponding height. Applying to each single CTT matrix element its closest simulated vertical profile, the CTH of the entire scene was estimated. To verify the improvement obtained by using WRF model simulated profiles, the CTH

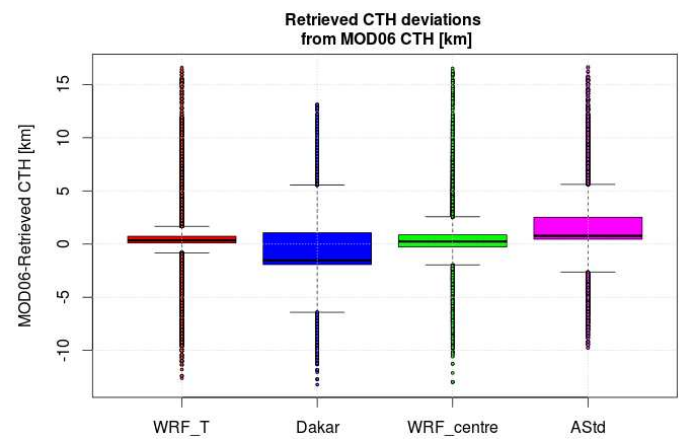

Fig. 5. Boxplot of the differences between MODIS CTH and the CTHs obtained applying different profiles to the MODIS CTT image: interpolated WRF profiles (WRF_T), Dakar radiosounding (Dakar), profile provided by WRF in the centre of the image (WRF_centre) and the U.S. Standard Atmosphere Model (AStd)

has been retrieved from the CTT provided by MODIS using temperature vertical profiles from: a) the U.S. Standard Atmosphere model, b) a nearby radiosounding (Dakar), c) the profiles provided by WRF interpolated to each pixel of the image and d) the profile provided by WRF in the centre of the image. Figure 5 shows the boxplot of the differences. The strong dependence of the estimated $\mathrm{CTH}$ on the chosen profile can be seen. Comparing the four different methods, it seems that WRF simulated temperature profiles applied pixel by pixel to the MODIS CTT image are the best compromise for CTH estimation. In this case, the differences between the results obtained with both WRF-based methods are included in the range $+/-1 \mathrm{~km}$. However, it is necessary to bear in mind that other scenarios can be more heterogeneous, in the sense that the vertical profiles can have more spatial variations. In those cases, the use of several vertical profiles would be even more appropriate. From now on, the method based on the application of the closest WRF simulated vertical temperature profile, for each single CTT matrix element will be used.

3) Data: To apply the radiative method to actual data, the MODIS scenes included in table I have been selected among the MODIS-Terra Cloud Product MOD06. All the images include liquid water and ice clouds, thin and thick clouds and low and high clouds (see the percentages in table I).

Since the MODIS IR spectral bands number 31 and 32 are quite similar to those of the JEM-EUSO IR camera, we can apply our method to the images corresponding to those bands to obtain the CTT and the CTH images. Then these images and the corresponding MODIS products are subtracted from each other to calculate the retrieved temperature and height differences. Also, the MODIS effective emissivity, thermodynamic phase, cloud top height and multi layer flag products can be used to analyse the results. It is worth noting that MODIS CTT retrieval uses a completely different methodology: BT in the 11- $\mu \mathrm{m}$ band for low clouds and the $\mathrm{CO}_{2}$-slicing method band $(13-15-\mu \mathrm{m})$ for the rest of clouds. This method, based on 4 bands $\left(\mathrm{CO}_{2}\right.$ channels), exploits the different sensitivity of each band to different heights, to retrieve the $\mathrm{CTH}$ of upper layer clouds very accurately (for more details see [35]). Even though MODIS has shown discrepancies with other sensors [36], we consider the difference between the CTT retrieved by 
TABLE I

PERCENTAGE OF PIXELS CLASSIFIED BY MODIS AS LIQUID WATER/ICE CLOUd AND THIN/THICK CLOUD. THE TABLE SHOWS THE DIVERSITY OF SITUATIONS STUDIED IN EACH SCENE.

\begin{tabular}{|c|c|c|c|c|c|c|}
\hline Image & Date & \multicolumn{2}{|c|}{ Liquid Water } & & Ice & Mixed/Unknown \\
\hline MOD06_L2.A2014092.1235 & $2^{\text {nd }}$ April 2014 & $53.0 \%$ & $\begin{array}{l}\text { Thick: } 75.0 \% \\
\text { Thin: } 25.0 \%\end{array}$ & $18.0 \%$ & $\begin{array}{l}\text { Thick: } 60.0 \% \\
\text { Thin: } 40.0 \%\end{array}$ & $29.0 \%$ \\
\hline MOD06_L2.A2010069.1215 & $10^{t h}$ March 2010 & $75.9 \%$ & $\begin{array}{c}\text { Thick: } 57.7 \% \\
\text { Thin: } 42.3 \%\end{array}$ & $13.7 \%$ & $\begin{array}{c}\text { Thick: } 82.2 \% \\
\text { Thin: } 17.8 \%\end{array}$ & $10.4 \%$ \\
\hline MOD06_L2.A2010085.1215 & $26^{t h}$ March 2010 & $88.0 \%$ & $\begin{array}{c}\text { Thick: } 83.8 \% \\
\text { Thin: } 16.2 \%\end{array}$ & $4.8 \%$ & $\begin{array}{c}\text { Thick: } 77.3 \% \\
\text { Thin: } 22.7 \%\end{array}$ & $7.2 \%$ \\
\hline MOD06_L2.A2010101.1215 & $11^{\text {th }}$ April 2010 & $65.0 \%$ & $\begin{array}{c}\text { Thick: } 66.8 \% \\
\text { Thin: } 32.2 \%\end{array}$ & $24.0 \%$ & $\begin{array}{c}\text { Thick: } 73.8 \% \\
\text { Thin: } 26.2 \%\end{array}$ & $11.0 \%$ \\
\hline
\end{tabular}

Thick $=\tau \geq 2$ Thin $=\tau<2$

our algorithm and the CTT given by MODIS as an indicator of the accuracy of the algorithm. Additionally to these scenarios, another set of images has been analyzed in the same way to check that the results of applying this methodology in other latitudes and seasons are similar, as explained in the introduction. The images of this new set also include very different clouds from the radiative point of view (liquid water/ ice phase, thin/thick and low/ high clouds) which allow us to extend the scope of the conclusions of this study.

\section{RESULTS}

\section{A. Stereo Vision Results}

The algorithm was tested on stereo pairs of satellite data from the geostationary MSG-2/MSG-3-SEVIRI, and the polar Envisat-AATSR. For these tests bi-spectral pairs were considered in bands different from those presented in [20] (10.8-12 $\mu \mathrm{m}$ and 11-12 $\mu \mathrm{m}$ for MSG and AATSR, respectively), and that are closer to those that will be used in the JEM-EUSO mission. The CTH retrieval was performed on parts of the scenes cited below, that were also in the FoV of MODIS, and the results are compared and commented in the following sections. The scene of $2^{\text {nd }}$ April 2014 at 12:35 UTC imaged by MODIS, was also observed in the same day by MSG-2 at 12:39 UTC and MSG-3 at 12:42 UTC. The three areas observed on $10^{\text {th }}$ March 2010 at 12:13 UTC, $11^{\text {th }}$ April 2010 at 12:19 UTC and $26^{\text {th }}$ March 2010 at 12:13 UTC by AATSR, partially overlap the scenes observed by MODIS at UTC 12:15 and described in table I. Three images at mid latitudes and at different seasons were also analyzed.

1) Experiments with MSG: A quantitative analysis of the results is shown in Figure 6. From the cumulative histogram it is clear that almost $80 \%$ of the error for the reconstructed heights is lower than $3 \mathrm{~km}$, that is, according to (3), well below half a pixel error in the disparity. The difference between the two height maps shows that the algorithm was able to retrieve the altitude of the highest clouds (above $8 \mathrm{~km}$ ), and for certain pixels the stereo results seem to overestimate the MODIS values. However, it should be noted that the data acquisition between the two systems is not synchronous, with a time lag of about 5 minutes. In addition, they have different pixel resolutions on the ground ( $3 \mathrm{~km}$ and $1 \mathrm{~km}$ at SSP for MSG and MODIS, respectively), and there are also differences in the original BT values, in particular for the highest clouds. The
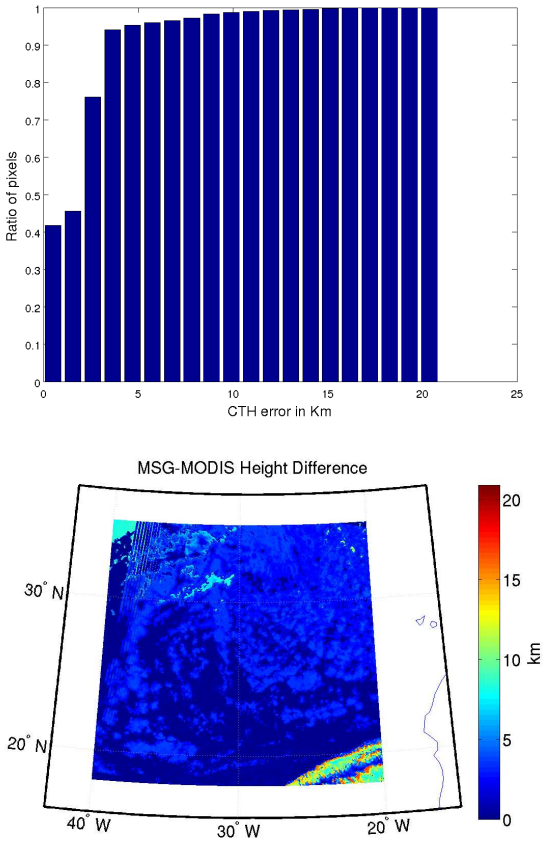

Fig. 6. MSG: Cumulative Histogram of the differences between stereo CTH and MODIS CTH (left); 2-D map of the differences (right).

low clouds up to those below $8 \mathrm{~km}$, are not detected at all. This is still in agreement with Equation (3), as disparities below 1 pixel might not be detected, and therefore not be distinguished from the ground. However this last result is not particularly meaningful for JEM-EUSO, as the final system will have a proper baseline and in addition, the algorithm applied to other sensors performs better, as discussed in the next section.

2) Experiments with AATSR: The stereo CTH estimated from the AATSR data were compared with the CTH available in the maps of MOD06 at $1 \mathrm{~km}$ of resolution. Before comparing the results of both sensors, AATSR and MODIS maps were resampled inside an area derived from the intersection of their field of views. The cumulative histogram in Figure 7 , of the differences among the retrieved stereo CTH and MODIS CTH shows that for the $26^{\text {th }}$ March 2010 scene, $\approx$ $70 \%$ of the pixels have an error $<700 \mathrm{~m}, \approx 90 \%$ of the pixels have an error $<1.5 \mathrm{~km}$. Similar results, only a little 


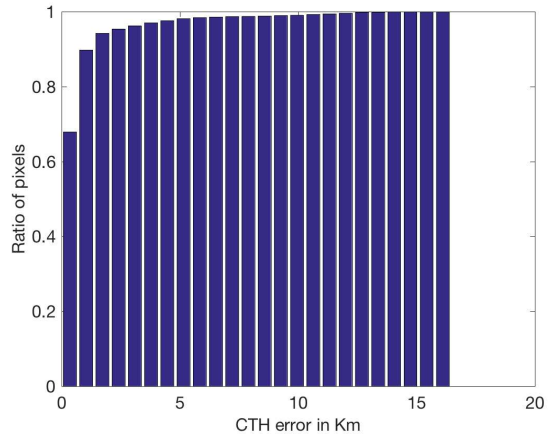

Fig. 7. AATSR: Cumulative Histogram of the differences between stereo CTH and MODIS CTH, for the scene $26^{t h}$ March 2010.

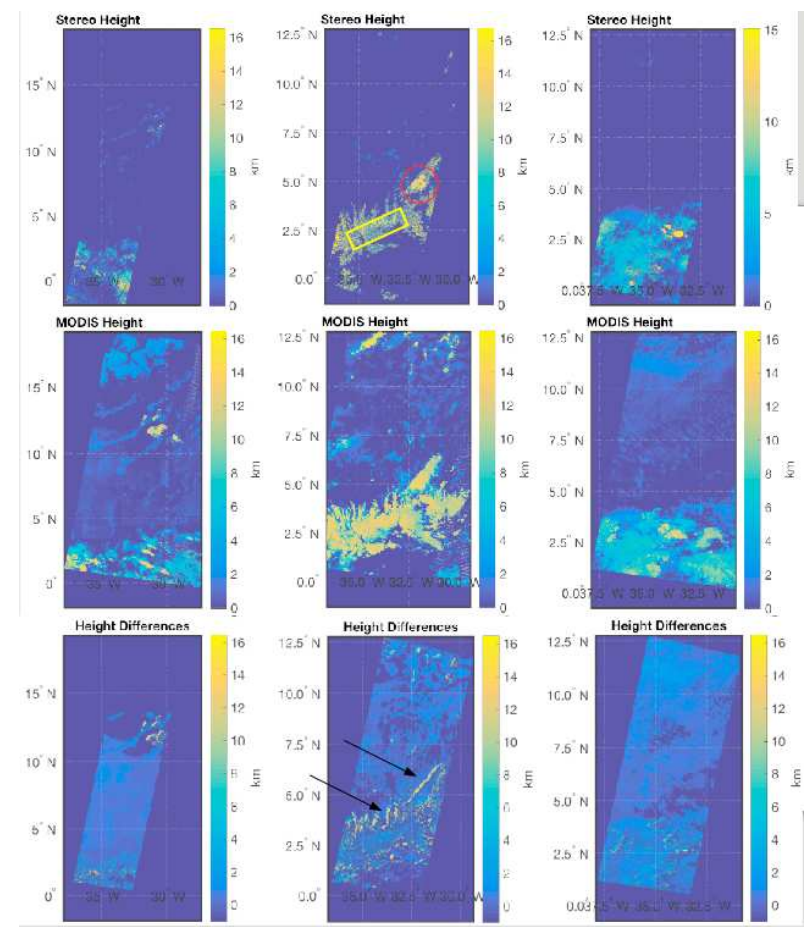

Fig. 8. AATSR 2-D maps of the retrieved stereo CTH (first row); MODIS 2-D maps of CTH (second row) and Differences of the previous maps (third row), for the scenes $26^{t h}$ March 2010, $11^{t h}$ April 2010 and $10^{t h}$ March 2010 (left to right). Note that the maps of the first and second rows also contain areas not belonging to the common parts of each scene.

worse than the previous, are obtained for the other scenes. These values are in agreement with the accuracy reachable by the AATSR instrument, according to the evaluation reported in section II-A2. Moreover, in Figure 8 it appears clear that the main structures of the stereo height maps reproduce the features of the MODIS maps, despite some issues regarding the lowest clouds (MODIS CTH $\tilde{<} 1.5 \mathrm{~km}$ ), and part of some highest clouds (MODIS CTH $\sim 10 \mathrm{~km}$ ). These findings are represented also in Figure 9 where the distribution of the errors is depicted, having the MODIS CTH as reference heights. In the case of heights $>5 \mathrm{~km}$ the error is more distributed. From the maps of the height differences (Figure 8, third row), it appears that apart from the lowest clouds, the altitudes of the highest clouds are in general well assigned. The errors are

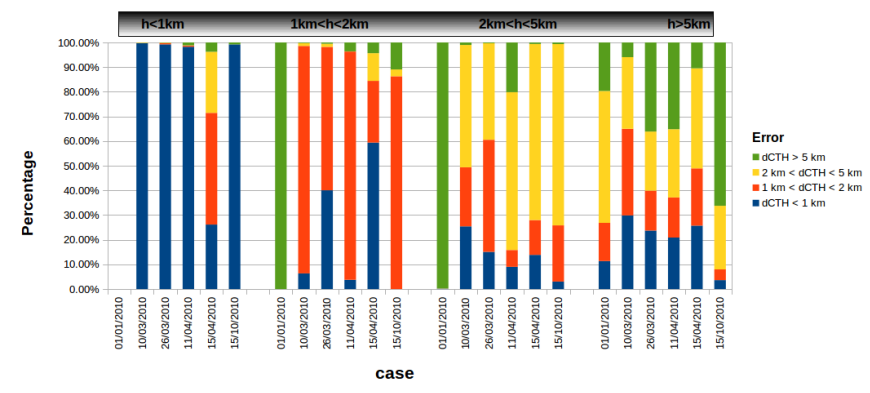

Fig. 9. AATSR: Distribution of the error between stereo CTH and MODIS $\mathrm{CTH}$, for very low $(\mathrm{CTH}<1 \mathrm{~km})$, low $(1 \mathrm{~km}<\mathrm{CTH}<2 \mathrm{~km})$, middle-height $(2 \mathrm{~km}<\mathrm{CTH}<5 \mathrm{~km})$ and high clouds $(\mathrm{CTH}>5 \mathrm{~km})$.

more concentrated on the edges, that result in underestimation compared to the MODIS CTH, or in the worst case, merging with the background. This is more evident in the $26^{t h}$ March 2010 and $11^{\text {th }}$ April 2010 scenes than in the $10^{\text {th }}$ March 2010 scene, where less than $10 \%$ of the pixels have a very high error. Moreover, for the second scene it is also evident that there are very high clouds whose stereo CTH agree with the MODIS CTH (highlighted by a circle in Figure 8), whereas in other cases the stereo $\mathrm{CTH}$ of the inner parts are lower than the MODIS CTH (highlighted by a rectangle in Figure 8).

It is important to highlight the influence on the results of some factors that are independent of the physics of the scenes. The time delay between two consecutive data acquisitions to obtain a stereo pair is $120 \mathrm{~s}$. This is a large delay, given the IFoV of the camera that contributes to obtain a reasonable baseline. However, the apparent motion on the images could not be attributed only to the parallax, but also to the natural motion of the clouds. In this case the recovered height should be corrected taking into account wind speed and direction. In this study, however, this effect was neglected, and the CTH was retrieved assuming a static scene. Another issue that must be taken into account, is the different native resolution of the two AATSR images. The forward view, has a lower resolution than the nadir $\left(1.5 \mathrm{~km} \times 2 \mathrm{~km}\right.$ and $1 \mathrm{~km}^{2}$ respectively, at the centre of the swaths), this leads to differences in the quality of both images when resampled, degrading the final match. Moreover the accuracy of around $\pm 2 \mathrm{~km}$ of the ATSR instrument geo-referencing ([13]) (assuming this true also for AATSR), could likely contribute to lower the outcome of the comparison against the reference height maps. This could reasonably explain most of the highest errors present on the edges of the highest clouds (Figure 8, third row areas pointed by the arrows) that, due to the mismatch between the AATSRMODIS maps, could be compared with lowest areas. Another source of error could also be the low emissivity of these pixels that gives rise to overestimated BT values. Despite the partial independence of this stereo algorithm from the BTs, in these cases the reconstruction seems to fail, although in other cases for pixels with comparable emissivity it performs better, as for the cloud within the circle in Figure 8, or leads to small error, as in the case of the cloud in the rectangle in Figure 8. But for this scene, it should also be considered that the compared 

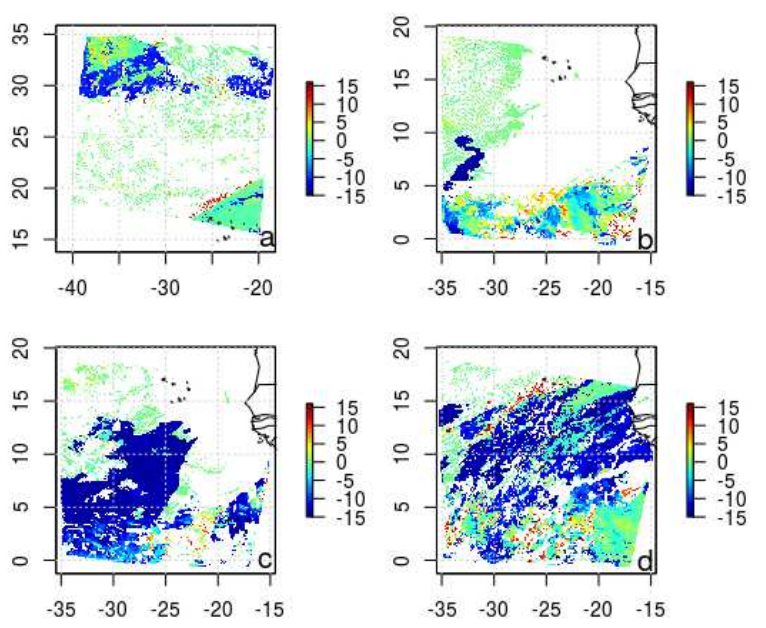

Fig. 10. Difference between MODIS CTH and WRF Cloud Fraction CTH [km] for the $2^{\text {nd }}$ April 2014 (a), $10^{\text {th }}$ March 2010 (b), 26 ${ }^{\text {th }}$ March 2010 (c) and $11^{\text {th }}$ April 2010 (d) analysed cases.

CTH maps differ 4 minutes in time and some differences in the cloud shapes can occur.

Finally the result of the CTH for the lowest clouds is conditioned by the impact that the instrument design has on the potential detectable disparity (II-A2).

In conclusion in the case of AATSR, it was possible to evaluate a larger range of heights than in the MSG case, due to a less problematic stereo configuration, despite that other elements affected the results.

\section{B. WRF Cloud Fraction Results}

The Cloud Fraction data were extracted from the simulation outputs described in section II-B1 and the resulting CTHs were compared with the respective MODIS ones. The deviation from MODIS CTHs (Figure 10) shows that the WRF Cloud Fraction method roughly reproduces the observed scenes. Low cloud cover due to local evaporation is, in general, reasonably well detected and the high clouds associated to large-scale motions can be recognized. Despite the fact that the model can reconstruct the cloudiness field on a broad scale, many pixel-by-pixel discrepancies between observed and simulated clouds are evident. Generally, the CTH retrieval is affected by the complexity of the meteorological condition, as for scales smaller than the synoptic one, it is difficult to make detailed predictions of atmospheric motions (see cases of $10^{\text {th }}$ March 2010 and $11^{\text {th }}$ April 2010). The highest errors are associated to medium-height clouds and to those middle-low clouds which WRF wrongly locates in the high troposphere (NW-NE corners of Fig. 10 a, S of Fig. 10 b, centre and SW of Fig. $10 \mathrm{c}$ and centre of Fig. $10 \mathrm{~d}$ ). Such inaccuracy in discerning low from very thin-high clouds combined with a general overestimation of the high-cloud cover may lead to errors higher than $5 \mathrm{~km}$, which strongly influence the entire model performance (Fig. 11 a). The analysis of some events occurred at medium latitudes in the four seasons of the year, confirms these results (Fig. 11 b).

Overall, the WRF model and the Cloud Fraction methodology seem to be able to qualitatively reproduce the cloudiness field, at least on a broad scale, although high errors between the observed (MODIS) and the WRF retrieved CTHs are found in the pixel-by-pixel comparison. Nevertheless, the model can provide reasonably good estimates of atmospheric temperature profile for each grid point of its inner domain, which can be successfully used to convert CTT in CTH, when necessary. Thus, despite that some uncertainties are still present, the use of a numerical model produces advantages and could be considered useful in this topic.

\section{Radiative Method Results}

The general procedure explained in section II-C, has been applied to the MODIS images shown in table I. To evaluate the performance of the procedure, the heights retrieved by the radiative method have been compared with the heights provided by MODIS. In Figure 12) the boxplot of the height differences corresponding to the four scenes is depicted. The corresponding IQRs are $0.83,0.95,1.48$ and 1.40 for $10^{\text {th }}$ and $26^{\text {th }}$ March 2010, $11^{\text {th }}$ April 2010 and $2^{\text {nd }}$ April 2014 respectively, which means that in the worst scenario $50 \%$ of the pixels have height differences lower than $1.5 \mathrm{~km}$, but in two of them $50 \%$ of the pixels have differences lower than $1 \mathrm{~km}$. To evaluate the results of the CTH retrieval process, the procedure is divided in two main steps: CTT and CTH retrievals.

1) CTT retrieval results: The CTT retrieval is a twostep procedure that calculates first the emissivity and then the temperature. Therefore these two processes have been evaluated separately.

The differences between the emissivity provided by MODIS and the emissivity retrieved by the LUT and the RTE algorithms, have been calculated for the scenarios in table I. The RTE algorithm retrieves the emissivity of high clouds very accurately. However the differences between the MODIS emissivity and the RTE emissivity are higher for low clouds. Figure 13(centre) is an example of this behaviour which is common for all the chosen images and was an expected result since the RTE approximation is only valid to clouds higher than $5 \mathrm{~km}$. The LUT emissivity shows a less bipolar pattern although, in general, it performs better for low clouds (see Figure 13(left)). The final emissivity used in the radiative method combines both algorithms to take advantage of both procedures (for example see Figure 13(right)).

When the SWA is applied to the images to retrieve the CTT, the results are very good for pixels with emissivity $\epsilon=1$. The medians vary from $-0.44 \mathrm{~km}\left(2^{\text {nd }}\right.$ April 2014) to $1.14 \mathrm{~K}\left(10^{\text {th }}\right.$ March 2010) and the corresponding IQR from $-0.88 \mathrm{~K}$ to $2.00 \mathrm{~K}$. However, the results are worse for $\epsilon<1$. Even though the median does not change significantly, the IQR increases notably. This effect increases as the emissivity decreases for all the scenarios studied, as expected. Figure 14 shows the differences between the MODIS CTT and SWA CTT (left) and the emissivity given by MODIS (right) for the scenario of $2^{\text {nd }}$ April 2014. The comparison between both images illustrates the good performance of the SWA for pixels with high emissivity and its difficulties to retrieve the CTT of 

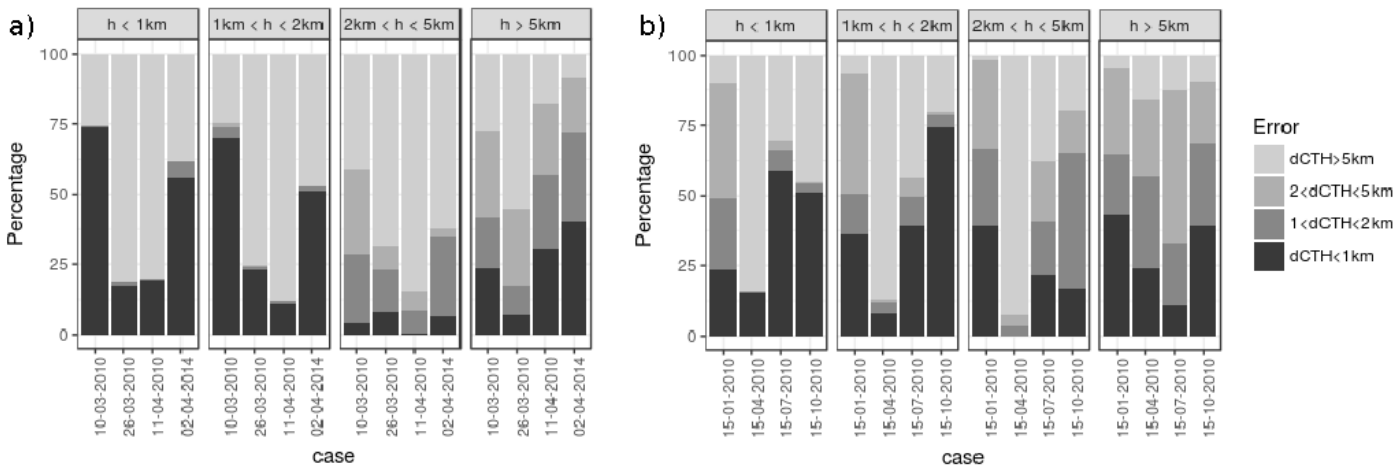

Fig. 11. Distribution of the error between MODIS and WRF CTH for very low (CTH $<1 \mathrm{~km})$, low $(1 \mathrm{~km}<\mathrm{CTH}<2 \mathrm{~km})$, middle-height $(2 \mathrm{~km}<\mathrm{CTH}<$ $5 \mathrm{~km})$ and high clouds $(\mathrm{CTH}>5 \mathrm{~km})$. Only clouds detected by both WRF and MODIS are considered.

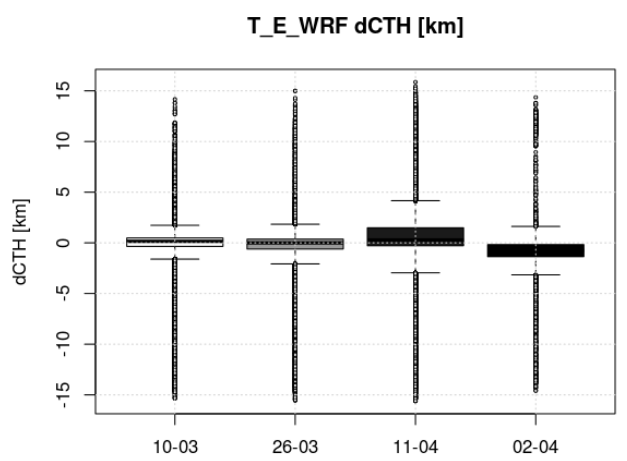

Fig. 12. Boxplot of the difference between the heights provided by MODIS and the heights calculated by the radiative method.

low emissivity pixels. The CTT retrievals performed by the LUT and RTE approximations show the same trend as the emissivities retrieved by this method, which means that the LUT approximation is more accurate to estimate the CTT in low clouds and the RTE approximation gives better results in high clouds. The median and the IQR of the final CTT results of the radiative method and the differences between the MODIS and the radiative method CTTs, are presented in table II. The values are similar for all the scenarios except for the $11^{\text {th }}$ April 2010 scene, where the high IQR and the low median temperature reveal the wide range of CTTs found and the high content of ice clouds which is in agreement with the values shown in table I. The IQR of the differences between the MODIS CTT and the radiative method CTT is between 1.93 and $2.55 \mathrm{~K}$ for the all scenarios except for the $11^{\text {th }}$ April 2010 scene, where it increases up to $4.43 \mathrm{~K}$.

In order to better evaluate these results, the pixels of each image have been grouped into three categories according to the emissivity: $\epsilon=1,0.5<=\epsilon<1$ and $\epsilon<0.5$. The statistic values have been recalculated for these categories. The corresponding medians do not follow a clear pattern however the IQR values increase remarkably for pixels with $\epsilon<0.5$, in particular for the $11^{\text {th }}$ April 2010 scenario, where the IQR reaches a value of $73 \mathrm{~K}$. These results demonstrate
TABLE II

STATISTIC VALUES OF THE CTT CALCULATED B Y THE RADIATIVE METHOD, AND DIFFERENCES BETWEEN THE CTTS PROVIDED BY MODIS AND THE RADIATIVE METHOD.

\begin{tabular}{|c|c|c|c|c|}
\hline Image & \multicolumn{2}{|c|}{ Radiat.Meth.CTT } & \multicolumn{2}{c|}{ CTT Differences } \\
\hline \hline & Median & IQR & Median & IQR \\
\hline \hline $2^{\text {nd }}$ April 2014 & 285.68 & 9.25 & -1.24 & 2.05 \\
\hline $10^{t h}$ March 2010 & 294.07 & 19.02 & 0.05 & 1.93 \\
\hline $26^{t h}$ March 2010 & 293.90 & 4.69 & 1.10 & 2.55 \\
\hline $11^{t h}$ April 2010 & 291.15 & 68.15 & 0.29 & 4.43 \\
\hline
\end{tabular}

that the main source of the differences are the clouds with $\epsilon<0.5$. This behaviour can be explained by two facts. First of all, the RTE approximation is only valid for clouds higher than $5 \mathrm{~km}$. Secondly, when the cloud emissivity is low, part of the radiance coming from the elements below the cloud (Earth's surface and atmosphere) passes through the cloud and contributes to the radiance received by the sensor which significantly affects the LUT emissivity retrievals, explaining the higher MODIS CTT and radiative method CTT differences for $\epsilon<0.5$. Also, the areas where the emissivity deviations are higher can be associated to areas of cloud superposition.

To evaluate the process of CTT retrieval from the emissivity independently of the accuracy of the emissivity, the CTT has been retrieved with the radiative method but using the emissivity provided by MODIS. An example of these differences can be found in Figure 15, where the image on the left corresponds to the differences between the MODIS CTT and the radiative method CTT for the $2^{\text {nd }}$ April 2014 scene, and the image on the right represents the differences between the MODIS CTT and the radiative method CTT using the MODIS emissivity, for the same scene. These last differences have been analysed for all the scenarios and the three categories above mentioned. The statistic values do not change dramatically except for the IQRs of pixels with $\epsilon<0.5$ for the $26^{\text {th }}$ March and $11^{\text {th }}$ April 2010 scenes. These results support the fact that the low-emissivity pixels are the main reason for the inaccuracies of the radiative method although the CTT retrieval process also contributes to the discrepancies, as can be seen in Figure 15 (right), especially in low emissivity pixels located at the edges of the 

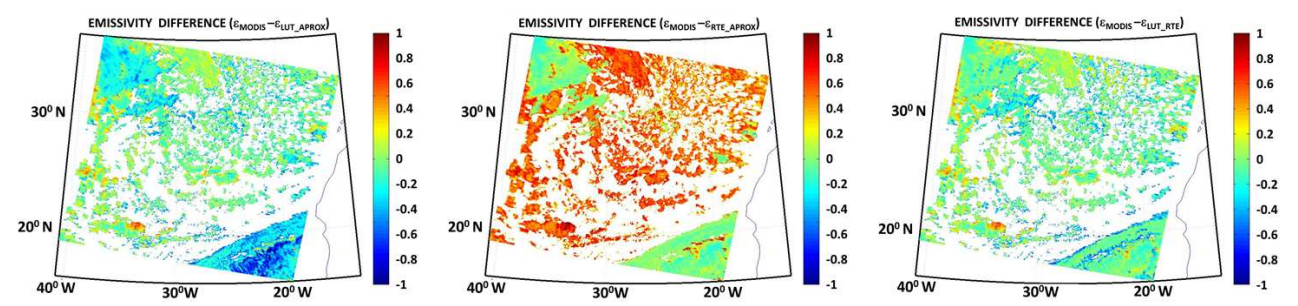

Fig. 13. Differences between the emissivity provided by MODIS and the emissivity calculated by the LUT approximation (left), the emissivity calculated by the RTE approximation (centre) and the emissivity calculated by the combined method used in the radiative method (right).
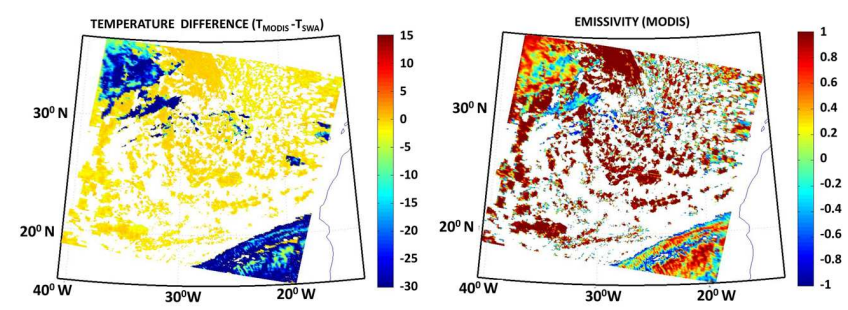

Fig. 14. Difference between the CTT provided by MODIS and the CTT calculated by the SWA (left). Emissivity provided by MODIS (right).
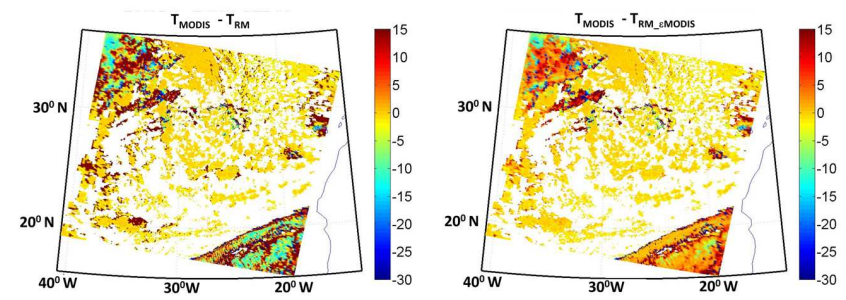

Fig. 15. Differences between the radiative method CTT and the MODIS CTT (left). Differences between the CTT retrieved by the radiative method but using the emissivity provided by MODIS and the MODIS CTT (right).

clouds. To check that these general results can be extended to other latitudes the procedure has been applied to a second set of images. The results are summarized in table III. The IQR for the differences between the $\mathrm{CTH}$ given by the radiative method and the CTH provided by MODIS are quite similar to the study cases analyzed previously. Although the median and the IQR for the CTT differences of these images are higher than for the previous set of images, the general conclusions are the same. The higher values correspond to images for which the IQRs of the CTT are also higher. The analysis of the CTT differences in the three emissivity categories confirms that this behaviour is related to a wide range of CTTs and the content of pixels with emissivity lower than 0.5 . More precisely, the percentage of pixels with $\epsilon<0.5$ in the $15^{\text {th }}$ July image is more than $29 \%$. In addition, in this image there is a big area where MODIS MLF (Multi Layer Flag) product remarks as superposition of clouds which contributes significantly to its high IQR value.

2) CTH retrieval results: To understand the influence on the $\mathrm{CTH}$ retrieval of the temperature-height conversion procedure

(already discussed in section II-C ) the difference between the CTH obtained by the radiative method and the CTH provided by MODIS was carried out. That difference has been compared with the difference between the $\mathrm{CTH}$ retrieved from the MODIS CTT and the CTH provided by MODIS directly. The heights were estimated applying the WRF temperature profile closest to each pixel of the CTT matrix. The boxplots corresponding to those differences are shown in Figure 16. It can be easily noticed that, even though the CTH retrieval with the profiles provided by WRF is the best option, this procedure also introduces a discrepancy in the radiative method since there are some differences between the CTH calculated with the radiative method and the MODIS emissivity, and the height provided directly by MODIS (right boxplot in Figure 16). Nevertheless the radiative method can produce reliable heights (left boxplot in Figure 16). For the other images referenced in table I, the results are similar. Summarizing, the results confirm that the low thick clouds are accurately retrieved by the SWA. The LUT and RTE approximations can be correctly applied to thin low clouds and thin high clouds respectively, whenever the emissivity is higher than 0.5 . The low emissivity is the main source of discrepancies although the CTT and the CTH retrieval processes also contribute to the total difference between the radiative method CTH and the MODIS CTH.

\section{CONClusions}

Two different stereo configurations of satellites were considered to try to recreate characteristics close to those of the JEMEUSO IR camera. First, a combination of two Meteosats were used, but the reconstruction was limited to a restricted range of heights. Secondly, the AATSR stereo system of Envisat allowed to test the method for a wider set of heights. The results are promising and support the use of the method in our case. Moreover the application of stereo on pairs composed 


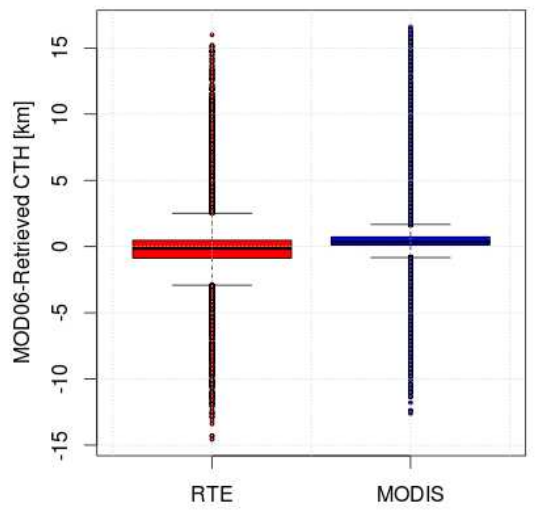

Fig. 16. Discrepancies from MODIS CTH and the CTHs obtained from the radiative method CTT (left) and from MODIS CTT (right). In both cases the temperatures were converted in heights using the WRF vertical profiles.

by images taken in different bands, instead of the more common use of one band, seemed not to affect the estimations substantially. The experiments showed an agreement between the obtained results and those expected according to the geometrical constraints. The better conditions of the JEMEUSO configuration, should improve the final reconstruction of most cloud types present in the FoV of JEM-EUSO.

The results of the radiative method reveal that the low thick clouds are accurately retrieved by the SWA, while the LUT and RTE approximations can be properly applied to the retrieval of thin clouds whenever the emissivity is higher than 0.5. The LUT provides better results for low clouds and the RTE for high clouds. The general procedure to calculate the CTT, indispensable to calculate the $\mathrm{CTH}$, is also very accurate. However, a deeper analysis of the results has revealed that the CTT retrieval, based on a previous estimation of the emissivity, is quite sensitive to emissivity errors. Even though the emissivity accuracy is good in general, small errors can be amplified in the CTT calculation. These errors are associated to very thin ice clouds. Nevertheless those pixels will be rejected before the application of the procedure. The final conclusion is that the radiative method proposed in this work would provide very accurate CTH information in most of the scenarios faced in JEM-EUSO mission, even though the constraints of the bi-spectral design of the IR camera is a strong handicap.

In the future, more work could be carried out with the aim to improve the accuracy of the emissivity estimation and reduce the sensitivity of the CTT retrieval to emissivity errors. The use of information provided by WRF could solve part of this inconvenience, although it would make the retrieval process less independent and automatic.

The usage of WRF model outputs can support the evaluation of the atmospheric conditions. First of all, its simulated vertical temperature profiles can be an advantageous tool for the temperature-height estimation, improving the $\mathrm{CTH}$ retrieval in radiative techniques. On the other hand, simulated atmospheric parameters can be used independently of IR observations to retrieve the CTH. In this work, the height estimation has been performed using only the cloud fraction output, even if many other parameters, such as the temperature, the dew point temperature, the relative humidity and the cloud hydrometric variables, available in all model vertical levels, may be used in a complementary way to evaluate the properties of the clouds.

In conclusion, all methodologies can be suitably used to retrieve the $\mathrm{CTH}$, exploiting both bi-spectral IR camera observations and data obtained with WRF model. To pursue the best performance in CTH estimation, these methods could be used in a complementary way, merging the potential of each one. The heights provided by the stereo vision method could be used as a first estimation to classify the clouds according to their height. Then, the emissivity calculated by the radiative method could provide a first classification of the clouds, according to their optical depth. Then for low and optically thick clouds, the CTH would be calculated by the radiative method, due to its high accuracy. For high and optically thin clouds $(\epsilon<0.5)$, where the radiative method is not able to retrieve the $\mathrm{CTH}$ with enough accuracy, the CTH would be provided by the stereo vision method. For clouds with emissivity between 0.5 and 1 , both methods can be applied. More work has to be done to better determine the accuracy of each method for each type of clouds in real scenarios. Through this approach, a deeper knowledge of the atmospheric conditions required by the JEM-EUSO mission will be attained. Moreover, the joint use of the three methods presented here could improve the accuracy of other interesting cloud parameters, such as the CTT and the emissivity. In the cloud types where the emissivity is too low to be retrieved by the radiative method accurately, the WRF information could be used to calculate the CTT from the CTH calculated with the stereo vision method, and from the CTT a more accurate value of emissivity can be obtained by using the RTE.

The methods presented in this paper have been tested using satellite data. More extensive tests will be performed analyzing the images that will be taken by the IR camera of the EUSO - Super Pressure Balloon2( [37]), which is an experiment on board a super pressure balloon for a flight of several weeks duration that it is expected to be launched in two years. On board the balloon an IR camera with similar characteristics to those foreseen for JEM-EUSO will be installed. Therefore the images of this camera will be the most appropriate to test the methodologies since they have been developed for this specific instrument and the JEM-EUSO mission. The results could be compared with those obtained by the satellite product repositories.

\section{ACKNOWLEDGEMENTS}

This work has been partially supported by the Italian Ministry of Foreign Affairs and International Cooperation. The authors of Universidad Carlos III de Madrid, want to acknowledge the Spanish Ministry MICINN for financial support of projects AYA2011-29489-C03-03 and AYA2012-39115-C0302 . The authors acknowledge the MODIS Science team for the Science Algorithms, the Processing Team for producing MODIS data, and the GES DAAC MODIS Data Support Team for making MODIS data available to the user community. We also thank EUMESAT for providing Meteosat data. The 
authors want also to thank the JEM-EUSO collaboration for the fruitful internal discussions.

\section{REFERENCES}

[1] A. Aab and et al.(Pierre Auger Coll.), "Trigger and aperture of the surface detector array of the Pierre Auger Observatory," Nuclear Instruments and Methods in Physics Research Section A: Accelerators, Spectrometers, Detectors and Associated Equipment, vol. A798, pp. 172-213, 2015.

[2] J. Abraham and et al.(Pierre Auger Coll.), "Trigger and aperture of the surface detector array of the Pierre Auger Observatory," Nuclear Instruments and Methods in Physics Research Section A: Accelerators, Spectrometers, Detectors and Associated Equipment, vol. A613, pp. 2939, 2010.

[3] J. H. Adams-Jr. and et al.(JEM-EUSO Coll.), "The JEM-EUSO instrument," Experimental Astronomy, vol. 40, no. 1, pp. 19-44, November 2015.

[4] T. Ebisuzaki and et al. (JEM-EUSO Coll.), "The JEM-EUSO mission," Advances in Space Research, vol. 53, no. 10, pp. 1499-1505, 2014

[5] J. H. Adams-Jr. and et al. (JEM-EUSO Collaboration), "The atmospheric monitoring system of the JEM-EUSO instrument," Experimental Astronomy, vol. 40, no. 1, pp. 45-60, November 2015.

[6] J. Adams-Jr. and et al. (JEM-EUSO Coll.), "The infrared camera onboard JEM-EUSO," Experimental Astronomy, vol. 40, no. 1, pp. 6189, November 2015.

[7] "MODIS (MODerate resolution Imaging Spectroradiometer) NASA." [Online]. Available: http://modis.gsfc.nasa.gov/

[8] "MODIS Cloud Optical Properties: User guide for the collection 6 level-2 MOD06/MYD06 Product and Associated Level-3 Datasets." [Online]. Available: https://modis-atmosphere.gsfc.nasa.gov/ sites/default/files/ModAtmo/C6MOD06OPUserGuide_1.pdf

[9] D. Lorenz, "Stereoscopic imaging from polar orbit and synthetic stereo imaging," Advances in Space Research, vol. 2, pp. 133-142, 1985.

[10] A. Prata and P. Turner, "Cloud-top height determination using ATSR data," Remote Sensing of Environment, vol. 59, pp. 1-13, 1997.

[11] C. Moroney, R. Davies, and J.-P. Muller, "Operational retrieval of cloudtop heights using MISR data," IEEE T. Geoscience and Remote Sensing, vol. 40, pp. 1532-1540, 2002.

[12] J.-P. Muller, M.-A. Denis, R. Dundas, K. Mitchell, C. Naud, and H. Mannstein, "Stereo cloud-top heights and cloud fraction retrieval from ATSR-2," International Journal of Remote Sensing, vol. 28, no. 9, p. 19211938, May 2007.

[13] G. Seiz, S. Tjemkes, and P. Watts, "Multiview cloud-top height and wind retrieval with photogrammetric methods: Application to Meteosat8 HRV observations," Journal of Applied Meteorology and Climatology, vol. 46, no. 8, pp. 1182-1195, 2007.

[14] R. Marchand and T. Ackerman, "An assessment of Multiangle Imaging Spectroradiometer (MISR) stereo-derived cloud top heights and cloud top winds using ground-based radar, lidar, and microwave radiometers," Journal of Geophysical Research, vol. 112, March 2007.

[15] D. Fisher, C. Poulsen, G. Thomas, and J.-P. Muller, "Synergy of stereo cloud top height and ORAC optimal estimation cloud retrieval: evaluation and application to AATSR," Atmospheric Measurement Techniques, vol. 9, pp. 909-928, 2016.

[16] E. Trucco and A. Verri, Introductory Techniques for 3-D Computer Vision. Upper Saddler River, New Jersey 07458: Prentice Hall, "1998".

[17] R. Szeliski, Computer Vision Algorithms and Applications. Springer, "2010".

[18] A. Anzalone, F. Isgrò, and et al.(JEM EUSO Coll.), "An experimental comparison of standard stereo matching algorithms applied to cloud top height estimation from satellite IR images," SPIE-Remote Sensing, Image and Signal Processing for Remote Sensing, vol. 10004, 2016.

[19] A. Anzalone and F. Isgrò, "A multi-spectral stereo method to retrieve cloud top height applied to geostationary satellite IR images," Int.Conf. on Computer Systems and Technologies, vol. 1164, 2016, pp. 1-4.

[20] K. Manizade, J. Spinhirne, and R. Lancaster, "Stereo cloud heights from multispectral IR imagery via region-of-interest segmentation," IEEE T. Geoscience and Remote Sensing, vol. 44, no. 9, pp. 2481-2491, 2006.

[21] D. Aminou, "MSG's SEVIRI instrument," ESA Bulletin(0376-4265), vol. 111, pp. 15-17, 2002

[22] D. Llewellyn-Jones, M. Edwards, C. Mutlow, A. Birks, I. Barton, and H. Tait, "AATSR: Global-Change and Surface-Temperature Measurements from Envisat," ESA Bulletin, no. 105, February 2001.
[23] W. Skamarock and et al., "A description of the advanced research WRF version 3," NCAR Technical Note, June 2008. [Online]. Available: http://www2.mmm.ucar.edu/wrf/users/docs/arw_v3.pdf

[24] E. Mlawer, S. Taubman, P. Brown, M. Iacono, and S. Clough, "Radiative transfer for inhomogeneous atmosphere: RRTM, a validated correlated-k model for the long-wave," Journal of Geophysical Research, vol. 102, pp. 16663-16682, 1997.

[25] J. Dudhia, "Numerical study of convection observed during the winter monsoon experiment using a mesoscale two-dimensional model," Journal of Atmospheric Science, vol. 46, pp. 3077-3107, 1989.

[26] S. Hong, Y. Noh, and J. Dudhia, "A new vertical diffusion package with an explicit treatment of entrainment processes," Monthly Weather Review, vol. 134, pp. 2318-2341, 2006.

[27] H. Morrison, G. Thompson, and V. Tatarskii, "Impact of cloud microphysics on the development of trailing stratiform precipitation in a simulated squall line: Comparison of one- and two-moment schemes," Monthly Weather Review, vol. 137, pp. 991-1007, 1997.

[28] Z. Wan and J. Dozier, "A generalized split-window algorithm for retrieving land-surface temperature from space," IEEE Transactions on Geoscience and Remote Sensing, vol. 34, no. 4, pp. 892-905, 1996.

[29] T. Inoue, "On the temperature and effective emissivity determination of semi-transparent cirrus clouds by bispectral measurements in the 10 micron window region," Journal of Meteorology Society Japan, vol. 63, pp. 88-99, 1985.

[30] K. Heidinger and M. Pavolonis, "Gazing at cirrus clouds for 25 years through a split window. Part I: Methodology." Journal of Applied Meteorology and Climatology, vol. 48, pp. 1100-1116, June 2009.

[31] S. Briz and et al., "Remote sensing of water clouds temperature with an infrared camera on board the International Space Station in the frame of Japan Experiment Module-Extreme Universe Space Observatory mission," Journal of Applied Remote Sensing, vol. 8, 2014.

[32] A. Berk and et al., "Band Model Method for Modeling Atmospheric Propagation at Arbitrarily Fine Spectral Resolution," U.S. Patent \# 7433806, 2008.

[33] S. Briz and et al., "Retrieval of optically thin cloud emissivity from brightness temperatures provided by IR camera of JEM-EUSO mission," European Physical Journal (EPJ) Web of Conferences, vol. 89, p. 03011, 2015.

[34] W. P. Menzel and et al., "Cloud top properties and cloud phase algorithm theoretical basis document," March 2013. [Online]. Available: http: //modis-atmos.gsfc.nasa.gov/_docs/MOD06_ATBD_2013_03_06.pdf

[35] - "MODIS global cloud-top pressure and amount estimation: Algorithm descriptions and results," Journal of Applied Meteorology and Climatology, vol. 47, pp. 1175-1198, 2008.

[36] I. Genkova, G. Seiz, P. Zuidema, G. Zhao, and L. D. Girolamo, "Cloud top height comparisons from ASTER, MISR and MODIS for trade wind cumuli," Remote Sensing of Environment, vol. 107, pp. 211-222, June 2007.

[37] "EUSO-SPB Mission and Instrument." [Online]. Available: http: //astroserve.mines.edu/euso_spb/2017-spb.html 